\title{
Video Article \\ Behavioral Phenotyping of Murine Disease Models with the Integrated Behavioral Station (INBEST)
}

\author{
Boris Sakic ${ }^{1}$, Marcella P. A. Cooper ${ }^{2}$, Sarah E. Taylor ${ }^{2}$, Milica Stojanovic ${ }^{2}$, Bosa Zagorac ${ }^{2}$, Minesh Kapadia ${ }^{3}$ \\ ${ }^{1}$ Department of Psychiatry and Behavioral Neurosciences, McMaster University \\ ${ }^{2}$ Department of Psychology, Neuroscience \& Behaviour, McMaster University \\ ${ }^{3}$ Neuroscience Program, McMaster University
}

Correspondence to: Boris Sakic at sakic@mcmaster.ca

URL: https://www.jove.com/video/51524

DOI: doi:10.3791/51524

Keywords: Behavior, Issue 98, behavioral phenotyping, Integrated Behavioral Station, disease models, home-cage monitoring, computerized tracking, chronic studies, mice

Date Published: 4/23/2015

Citation: Sakic, B., Cooper, M.P., Taylor, S.E., Stojanovic, M., Zagorac, B., Kapadia, M. Behavioral Phenotyping of Murine Disease Models with the Integrated Behavioral Station (INBEST). J. Vis. Exp. (98), e51524, doi:10.3791/51524 (2015).

\section{Abstract}

Due to rapid advances in genetic engineering, small rodents have become the preferred subjects in many disciplines of biomedical research. In studies of chronic CNS disorders, there is an increasing demand for murine models with high validity at the behavioral level. However, multiple pathogenic mechanisms and complex functional deficits often impose challenges to reliably measure and interpret behavior of chronically sick mice. Therefore, the assessment of peripheral pathology and a behavioral profile at several time points using a battery of tests are required. Video-tracking, behavioral spectroscopy, and remote acquisition of physiological measures are emerging technologies that allow for comprehensive, accurate, and unbiased behavioral analysis in a home-base-like setting. This report describes a refined phenotyping protocol, which includes a custom-made monitoring apparatus (Integrated Behavioral Station, INBEST) that focuses on prolonged measurements of basic functional outputs, such as spontaneous activity, food/water intake and motivated behavior in a relatively stress-free environment. Technical and conceptual improvements in INBEST design may further promote reproducibility and standardization of behavioral studies.

\section{Video Link}

The video component of this article can be found at https://www.jove.com/video/51524/

\section{Introduction}

Rapid advancements in genetic engineering over the past few decades have led to an unprecedented proliferation of animal models of human diseases. Mice have gained the status of primary experimental subjects in biomedical sciences for several reasons. From a practical standpoint, they have a high reproduction rate, are relatively inexpensive, and are easy to house. From a conceptual point of view, they are genetically close to humans, can be genetically modified with relative ease, and have highly-developed endocrine, immune, and nervous systems. In addition to the lesions at genetic and cellular levels, contemporary studies of brain disorders require the demonstration of replicable functional deficits that highlight face, construct, or predictive validity of a new mouse model ${ }^{1}$.

An acute infection in a homoeothermic mammal often results in the febrile response, which together with sickness behavior, constitutes one of the principal survival mechanisms ${ }^{2}$. Acutely sick animals display significant alterations in food/water intake and performance in tasks reflective of emotional reactivity, exploratory behavior, and learning/memory capacity. These changes largely account for impaired social/sexual activity and preservation of energy for defensive immune reactions. However, when acute conditions turn chronic (as seen in many immunological, endocrine and neurological diseases), behavioral performance can further deteriorate due to structural damage of various organs, including the brain ${ }^{3}$.

Human and animal neurodegenerative diseases are often accompanied by a constellation of neurological and behavioral deficits. Therefore, a key purpose in behavioral studies of chronically sick animals is to distinguish central effects from the deficits induced by peripheral symptomatology. However, the relatively short duration of standard behavioral tasks limits the collection of information concerning basic functional measures, such as olfaction, resting, sleep, food/water intake, or epileptic episodes. Inclusion of these measures improves behavioral profiling and permits better interpretation of performance in activity-demanding tasks.

\section{Refinements in behavioral phenotyping of diseased mice}

The inadequacies in assessing the behavioral profile of sick mice have necessitated continuous monitoring of singly-housed mice by fastprocessing PCs. Although diverse behavioral batteries can be designed ${ }^{4,5}$, listed below are the procedures that have been used to successfully establish an animal model of neuropsychiatric lupus ${ }^{6}$. This battery is repeatedly applied in both sub-chronic and chronic models of disease (Figure 1), such as mild cognitive impairment and Alzheimer's disease ${ }^{7}$. Following a series of neurological tests ${ }^{8-10}$, a custom-made apparatus, designed to meet the above demands by utilizing continuous monitoring of multiple behavioral outputs in an enriched home-cagelike environment, can be employed. Such an ethologically-based approach to the assessment of spontaneous exploratory activity and motivated 
behavior provides a more comprehensive understanding of performance deficits in other paradigms, such as those reflective of learning and memory.

\section{Level of Anticipated Stress}

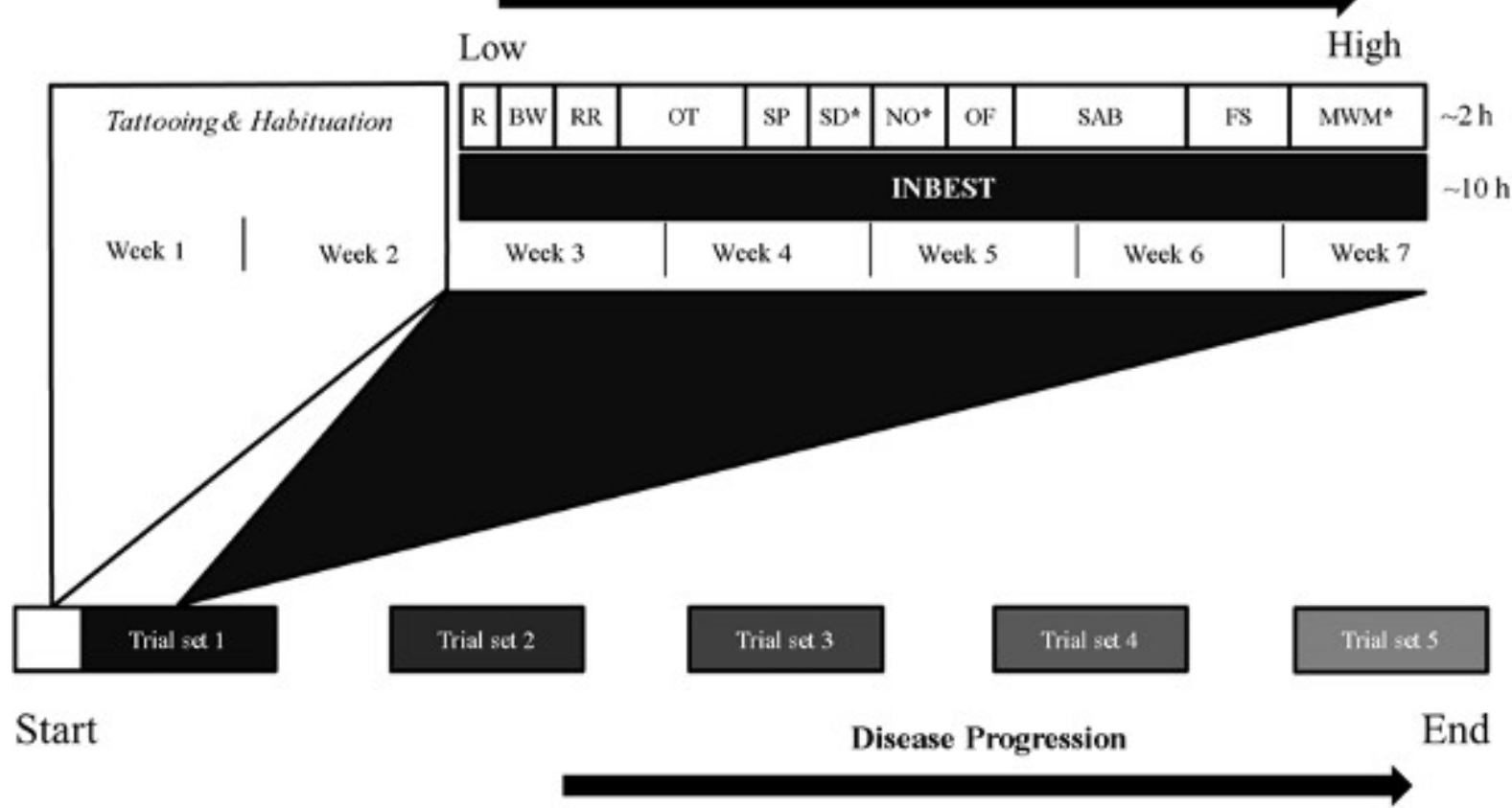

Figure 1. Schematic representation of longitudinal behavioral phenotyping in our laboratory. The behavioral battery is designed to evolve from less- towards more- stressful tasks, which are repeated at different time points to assess the effects of sustained factors such as disease progression, pharmacological treatment, or immunological responses. INBEST and individual tests are performed during the dark phase, often over 10 and $2 \mathrm{hr}$, respectively. Abbreviations: R - reflexes; BW - Beam Walking test; RR - Rotarod; OT - Olfactory tests; SP - Sucrose Preference test; SD - Step Down test; NO - Novel Object test; OF - Open-field test; SAB - Spontaneous Alternation Behavior; FS - Forced Swim test; MWM - Morris Water Maze. * - aspects of the test (e.g., location, context, color, shape) that need to be altered in subsequent trials throughout course of experiment.

Continuous video-recording and analysis of behavior in a home-cage-like environment were first reported in $2007^{11}$. A more complex automated apparatus that integrates behavioral tests used in studies with autoimmune mice was presented at the 'Measuring Behavior' meeting one year later ${ }^{12}$. The Integrated Behavioral Station (INBEST, Figure 2A) is a modular system, which comprises of a shelter, computer controlled light stimulus, two photocell-controlled lickometers (one for water, one for a solution of interest), an automated food dispenser, a computerized running wheel, and a digitized climbing mesh. Latencies, frequencies, and durations of specific behaviors are examined using customized software. Locomotor and exploratory activity (e.g., of a novel object or an unfamiliar conspecific) can be assessed with video-tracking software (List of Materials/Equipment), while sleeping and less frequent behavioral patterns, such as self-injurious behavior and seizures, can be scored manually with video-tracking software or dedicated event-recording packages. Eight complete INBEST/video setups are used, thus allowing concurrent monitoring of 4 experimental and 4 control animals (Figure 2B). 


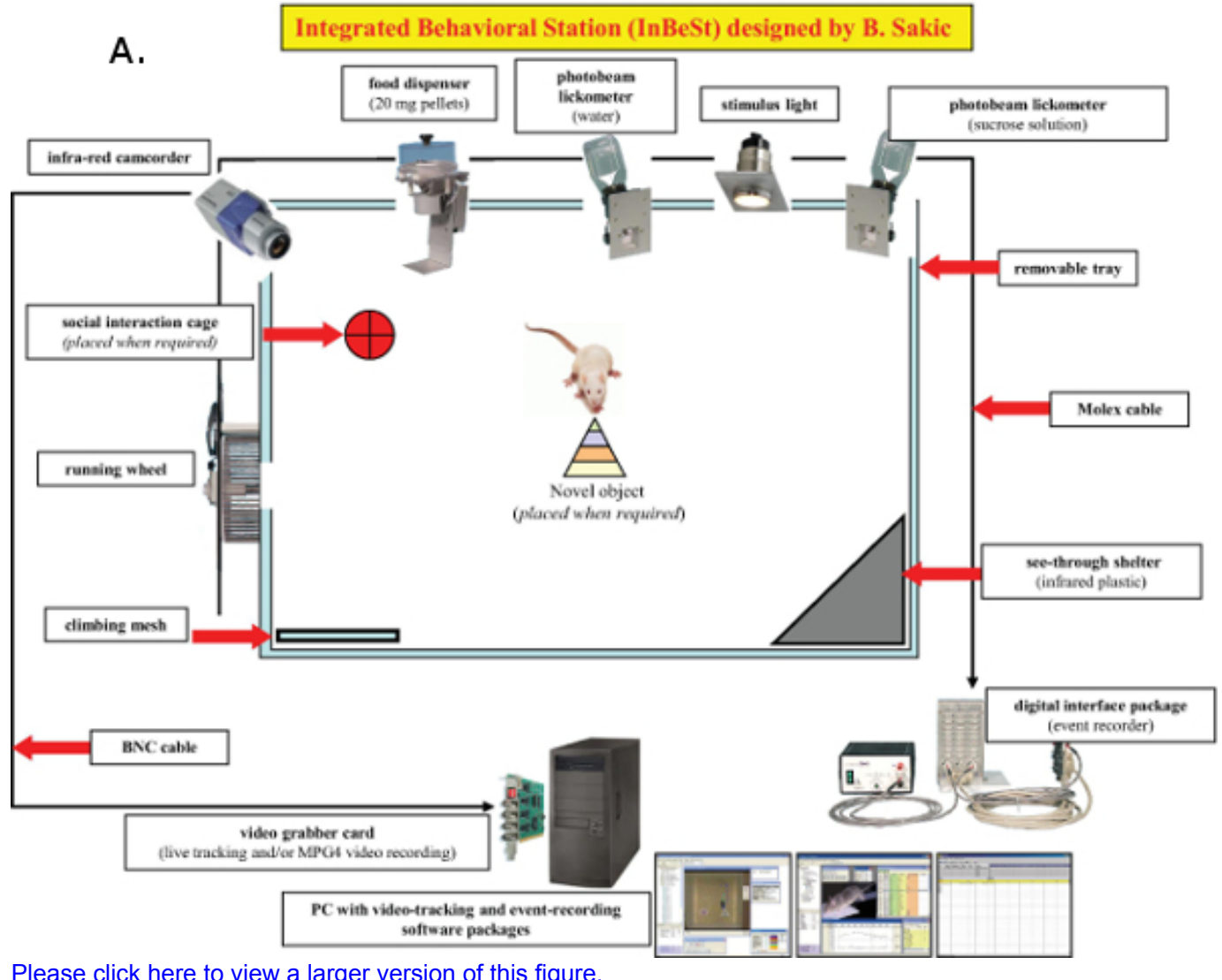

Please click here to view a larger version of this figure.

B.

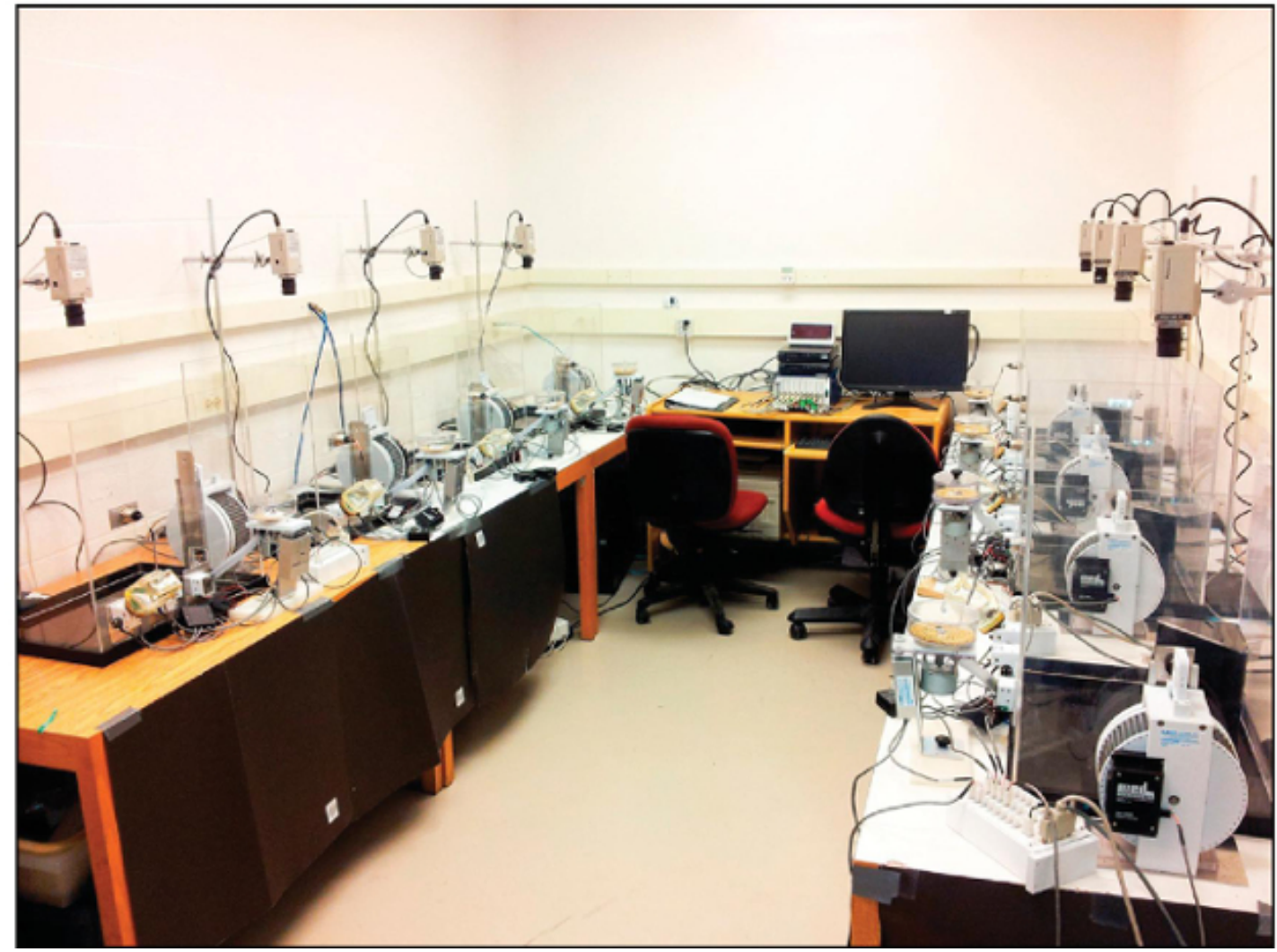

Figure 2. Integrated Behavioral Station. (A) Schematic representation of hardware, and software used in designing an INBEST box $(L=39 x$ $\mathrm{W}=53 \mathrm{xH}=50 \mathrm{~cm}$ ). (B) Eight complete INBEST boxes provide an opportunity for concurrent home-cage monitoring of four experimental and four control mice. 
Dependent variables include measurements of food/water intake, responsiveness to palatable stimulation, spontaneous ambulatory activity, climbing, voluntary running, anxiety-related behaviors (e.g., exploration of novel object), grooming, seizing and sleeping. Additionally, visual stimuli can be presented for conditioning and learning paradigms. The advantages of INBEST over standard behavioral testing include the elimination of confounding effects induced by transportation stress, as well as continuous, automated collection of measures reflective of nocturnal activity, exploration, anxiety-related and depressive-like behaviors. The integration of sensitive hardware components with a videotracking package yields a wealth of information, which permits improved assessment of behavior in relation to progression of chronic disease in diverse animal models. INBEST can be used to study other chronic CNS disorders (e.g., autism, major depression, schizophrenia), as well as in longitudinal studies focusing on neurodevelopment, behavioral effects of systemic / neoplastic disorders, and prolonged pharmacotherapy.

\section{Protocol}

All procedures are approved by the McMaster University Animal Care Committee and performed in accordance to guidelines set out by the Canadian Council of Animal Care.

\section{General Procedures}

1. Habituate mice for $1-2$ weeks to an existing $12 \mathrm{hr}$ light/dark cycle (e.g., $8 \mathrm{AM}-8 \mathrm{PM})$. Perform all procedures and testing during the dark cycle, with RT, humidity and light intensity being relatively constant.

2. Mark or tail-tattoo all mice for easy, numerical identification over a prolonged period and handle them 1-2 hr daily over 5-7 days.

3. Repeat daily measurements of rectal temperature, body weight and food/water intake to detect potential fever and/or malnutrition induced by aging or disease progress. Standard exclusion criteria include low body weight due to reduced food/water intake, hunched posture with ruffled fur, hydrocephalus, porphyrin discharge around eyes, etc.

4. To identify neurological deficits that may confound overall activity and performance, perform standard sensorimotor tests such as the hind limb clasping reflex ${ }^{13}$, visual placing reflex ${ }^{14}$, geotaxis test ${ }^{15}$, basket test ${ }^{16}$, beam walking test ${ }^{17-19}$, Rotarod ${ }^{20}$, and olfactory tests ${ }^{21}$. Note: The results may also help in correlational analysis with INBEST measures, more careful selection of other procedures (e.g., Morris water maze if mice are blind, novel object test if mice are hyposmic/anosmic), reduction of within-group variability, and exclusion of mice with birth deficits or infection.

5. Clean the plastic and glass apparatus with disinfectant to remove urinary trails while testing mice from experimental and control groups in an alternating fashion.

\section{Integrated Behavioral Station (INBEST) Procedure}

1. Home-cage set up

1. Fill food dispensers with $20 \mathrm{mg}$ mouse chow pellets.

2. Fill bottles with tap water.

Note: A second bottle can be filled with a solution of interest, such as sucrose or saccharine solution for a preference test.

3. Weigh bottles to calculate the volume consumed at the end of the session.

4. Insert bottle spouts into the lickometers. Make sure the nozzle does not block the infrared sensor; if it does, reduce the length of the spout.

5. Place shelters in the selected corner of the home-cage.

2. Computer set up

Note: The software commands provided in steps 2.2-2.11 are relevant to the Ethovision XT 8.5 software package (specified in the List of Materials/Equipment) and testing conditions in our laboratory.

1. Illuminate the room with diffuse, dim light that is sufficient for video-tracking, but does not reflect off the box, floor or walls.

2. Open a default video-tracking project and set up the Experiment Settings, by keying in relevant details (e.g., date/time of study, group assignment, room conditions etc.). Subsequently, choose the appropriate video source (Picolo Diligent grabber), number of arenas (4), tracking points (center-, nose- and tail-point), and units of measurement (centimeters, seconds, and degrees).

3. After selecting the Trial List tab under Setup, define the number of trials by clicking the Add Trials button (1). Next, specify independent variables (e.g., mouse ID, gender, group assignment, strain) using the Add Variable button.

4. Click the Arena Settings tab and capture the background image from the live video. Define the parameters of the individual arena by outlining the outer perimeter using the appropriate drawing tool (such as create rectangle/polyline/ellipse).

5. Next, add zones of interest by clicking the Add Zone group button and outlining the various zones (e.g., floor, lickometers, food dispenser, climbing mesh etc.) in an identical manner. Add hidden zones for the areas where the mouse cannot be seen (e.g., shelter and running wheel, Figure 2) by clicking the Add Hidden Zone Group button. Ensure that an entry/exit is specified and linked to each hidden zone.

6. Repeat steps 2.4-2.5 for each arena. Perform arena calibration by highlighting Calibration and using the appropriate tool (create calibration scale/axes) to provide arena width and length. Lastly, validate Arena settings by clicking the Validate Arena Settings button.

7. Highlight the Trial Control Settings tab and specify start / stop conditions and trial length. Set the start condition to begin when the duration of the center-point exceeds $1 \mathrm{sec}$ in the arena. Manipulate the trial duration by expanding the stop condition box and setting the trial to terminate after a delay, such as $10 \mathrm{hr}$.

8. Under the Detection Settings tab, highlight the appropriate detection methods (e.g., dynamic subtraction and model-based).

9. Next, grab the reference image of the empty arena by clicking the Settings button in the Detection tab and pressing the Grab Current button.

10. Adjust the range of contrast such that the center, nose and tail-based detection for each mouse are reliable, accurate and continuous For albino mice, specify that the mouse is brighter than the background, and darker than the background if using a pigmented strain. Note: The subject size and video sample rate can be changed according to the distance between the overhead camera and the subject, as well as the processing speed of the PC used (e.g., 14.9 frames/sec). 
11. Ensure all changes are saved before exiting the detection settings module.

12. Turn on the interface device, which is responsible for converting recorded analog events from input devices (e.g., interruption of infrared beam, movement of the running wheel etc.) into digital logs.

3. Data Acquisition

Note: The following software commands are relevant to the custom-made Med PC IV routine ("wizard") that provides step-by-step input of session parameters (e.g., $10 \mathrm{hr}$ trial duration, mouse ID, group assignment etc.)

1. Place each mouse into the assigned box.

2. Synchronize video- and event-tracking packages by simultaneously pressing the 'record' buttons.

3. Leave the experimental room quietly.

4. When the recording period expires (e.g., few hours, day, or weeks), remove mice and return them to their home-cages.

5. Measure bottle weights and save all digital recordings on digital media (hard drive, portable USB stick, DVD).

6. Transfer raw data to a spreadsheet.

7. Save the MPG files for subsequent scoring of infrequent behavioral acts (e.g., stereotypy, seizures).

\section{Representative Results}

Figure 3 exemplifies different read-outs in a prolonged behavioral study with CD1 mice. Data represents baseline performance (days 6 to 2 prior to surgery), post-surgery recovery (days 2 to 4 ) and behavioral effects induced by sustained intra-cerebro-ventricular administration of brain-reactive antibodies (days 6 to 10; where 0 denotes the surgery day). Analysis with event-recording software reveals that the experimental group displays impairments in ingestive behavior, as evidenced by a lower frequency of water bottle licks (A), increased latency to approach the sucrose solution (B), and decreased food consumption (C) during the experimental period. Coinciding with these changes, they also show diminished running wheel activity in comparison to control mice (D). As measured by video-tracking software, the experimental group also ambulates less in the home-cage $(E)$ and prefers to spend more time in the shelter $(F)$. These behavioral differences are illustrated on sample ethograms $(G)$. 

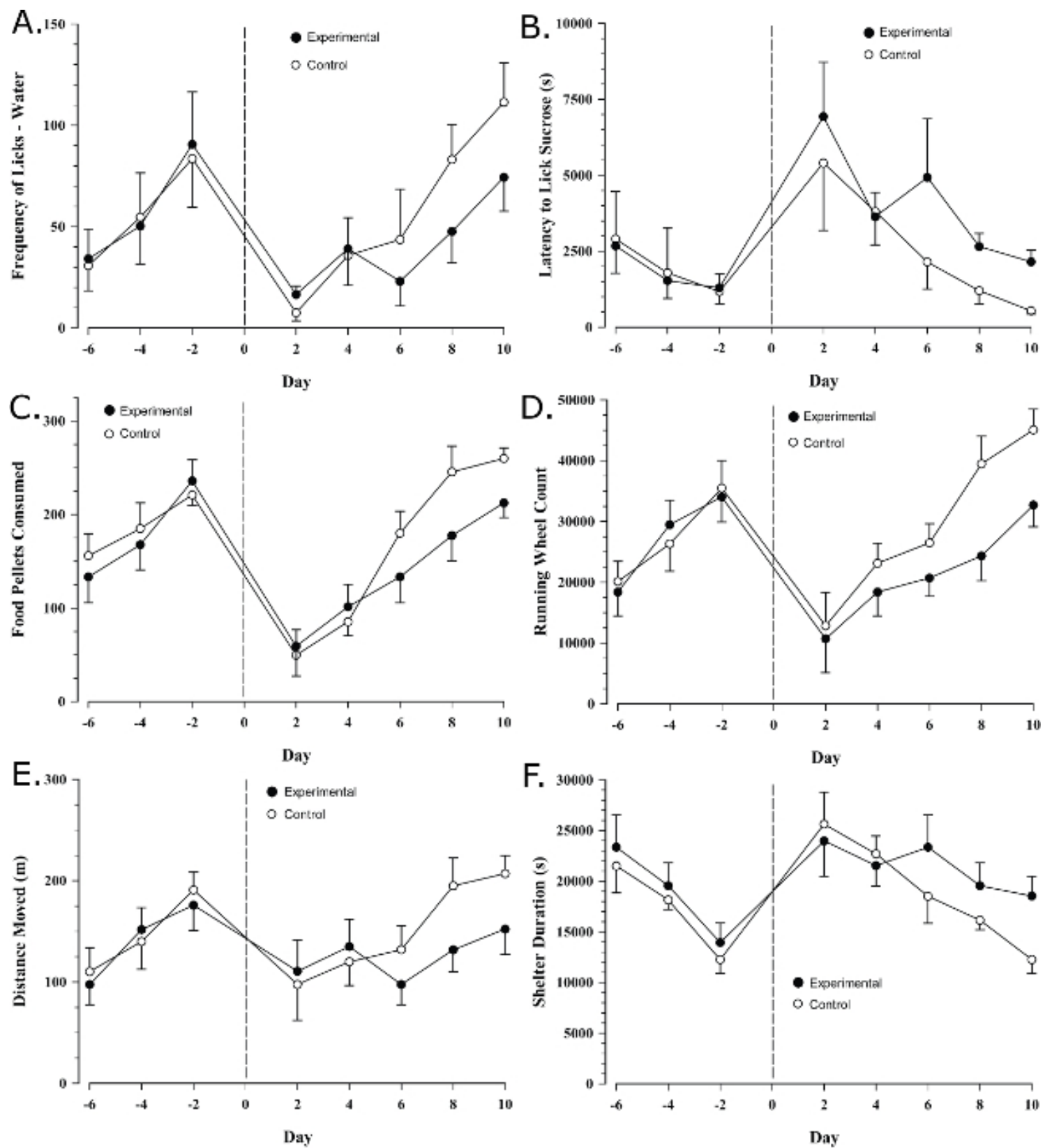

Please click here to view a larger version of this figure.

G.

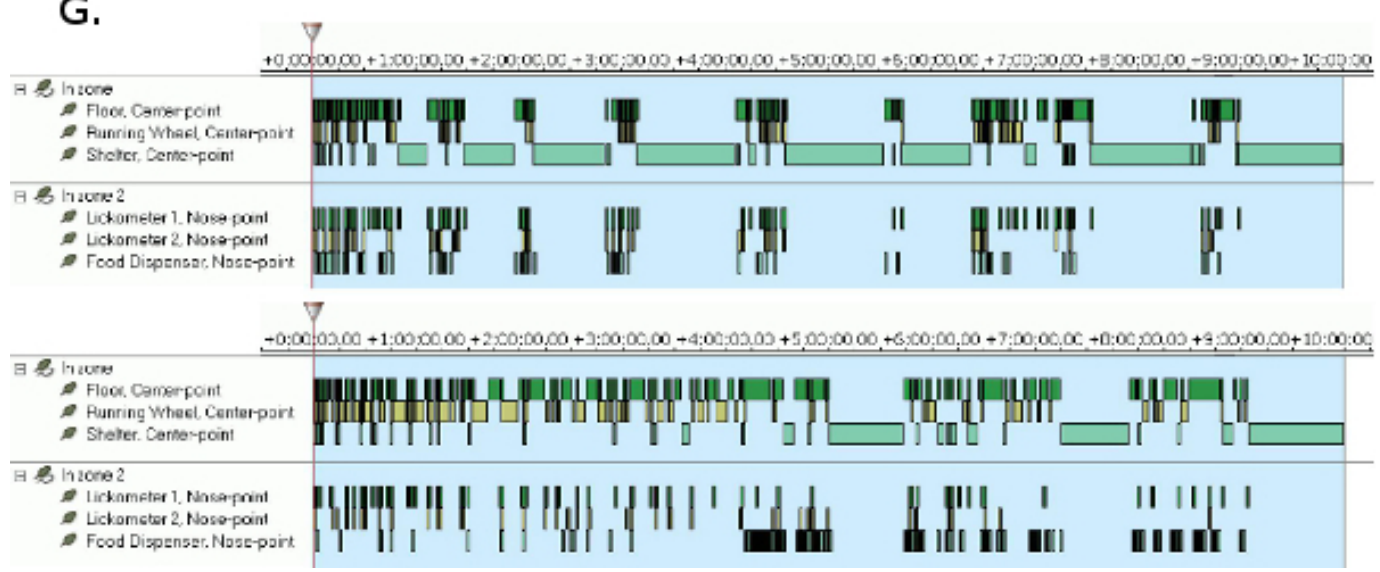

Please click here to view a larger version of this figure. 
Figure 3. Representative variables in a series of 10-hr daily sessions illustrating the discriminant power of the INBEST system. Experimental mice (exposed to brain-reactive antibodies over 2 weeks) drink less water (A), take longer to approach sucrose solution (B), and consume less food (C) during the testing period. Coinciding with these changes, they also show impaired activity, as exemplified by reduced running wheel count (D), decreased ambulation $(E)$ and prolonged stay in the shelter $(\mathbf{F})$. These behavioral differences are illustrated on sample ethograms (G). The top panel displays the behavior of an experimental mouse on day 6 , characterized by reduced ingestive behavior, lower running wheel activity and increased shelter time in comparison to a control mouse receiving a vehicle (bottom panel).

\section{Discussion}

The detection of functional effects in animals largely depends on the researcher's ability to limit the variability inherent to behavioral studies. Therefore, it is important to meticulously control and minimize potential confounds that may reduce the reliability and reproducibility of behavioral data. At the same time, it is important to recognize that no test reflects a single domain of behavior, that knowledge of neurological function is mandatory, and that behavior is highly sensitive to external stressors. If the above postulates are appreciated, one may conclude that comprehensive behavioral analysis should include the time course of the response measured, as well as, involve basic functional traits and paradigms that tap into specific behavioral aspects. Many of these criteria can be fulfilled using computerized assessment of movements and behavioral acts in an enriched home-cage environment.

Thus far, it has been emphasized that behavioral phenotyping of murine models of human disease warrants additional considerations. This notion is based on the premise that functional homeostasis is challenged by internal and external stressors during disease onset. Although all potential confounds may not be eliminated by the introduction of automated, home-cage phenotyping, issues relating to inconsistent environmental settings, transportation stress, and repeated handling are minimized. This significantly enhances consistency and precision across studies; even small reductions in variability can improve detection of the effects induced by an incipient disease. Indeed, INBEST provides a wealth of information, which permits more accurate assessment of the onset, kinetics, and severity of behavioral changes, as well as important relationships among various disease-induced behavioral deficits. Reliable video-tracking depends on two lighting conditions. First, diffuse light is required in the testing room to prevent artifacts from nearby reflecting objects. Second, high color contrast can be achieved by choosing an appropriate floor color to differ from the subject color as much as possible. In our lab, this is achieved by using flood-lights positioned below the INBEST boxes and black floor trays when monitoring albino mice (white or gray background would be adequate if testing pigmented strains). With respect to the event recording aspect of INBEST, the current hardware setting (1 Piccolo video card with 4 inputs) limits 4 boxes to be concurrently used per PC. This is a rather small number of boxes, while a more suitable set-up would require 8 or even 16 cages, and thus 2 or 4 PCs, respectively. Preferably, INBEST can be used continuously over 24 -hr as the home-cage. This would allow animals to habituate fully to the environment and establish stable, circadian behavioral patterns, which can be analyzed in an unbiased manner. To prevent computer data loss due to power outage, a continuous power supply (or at least an uninterruptible power source) must be secured. Lastly, to ensure proper assessment of daily food intake, it should be noted that the size of food pellets should not exceed the size of the holes in the food dispenser (the recommended size of a single food pellet is $20 \mathrm{mg}$ ).

It is not to be overlooked, however, that this analysis should also incorporate how different INBEST measures may interact with each other. For example, mice that spend more time in the running wheel are likely to ingest higher quantities of food and water to meet their increased caloric demands. Similarly, mice ingesting more sucrose solution may decrease their intake of food. The interpretation of these results may further be complicated by the general improvement of performance over time, particularly in regards to ingestive behavior and running wheel activity. Given their incentive-properties, experimenters may also consider limiting access to sucrose solution and the running wheel to counteract the likelihood of post-ingestive effects and excessive weight loss, respectively. However, these concerns may be more relevant in some strains than others because different strains of mice have dissimilar behavioral profiles. Although performing both baseline and experimental assessment controls for many of the issues above, experimenters need to recognize that these variables need to be taken into account when interpreting INBEST data. At the same time, some aspects of behavior cannot be studied in the home-cage environment, thus necessitating a combination with standard tests to complete the behavioral profile of subjects.

Computerized monitoring within standardized, but flexible environments appears to be the logical next step in contemporary behavioral analysis. Such a non-invasive, ethologically-based approach will allow researchers to observe the full repertoire of behavioral responses over a prolonged period of time. Theoretically, this can be accomplished by studying behavior in a "virtual", enriched environment that closely resembles a natural habitat. Several research groups have described vision-based tracking tools that support behavioral phenotyping of mice in their home-cage ${ }^{22-25}$, in dyads ${ }^{26,27}$, or in the context of large social groups ${ }^{28}$. High precision and spatial resolution can be achieved by integrating videotracking with microchip technology for simultaneous and synchronized collection of behavioral data in a group of mice ${ }^{28}$. Thermographic cameras capable of detecting heat-signatures can be combined with implantable microchips or transponders to provide the relative location and basic physiological functions of each mouse (e.g., body temperature, heart/breathing rate). In addition, an advanced 3D tracking system would produce more accurate and quantitative recognition of behavioral acts. In order to repeatedly run a variety of tests, such a system should be automated, remotely-controlled, and modular. For example, spatial memory can be studied in larger environments by programming the appearance of distal cues on LCD walls, or by presenting/hiding dispensers with palatable food from movable floors. In a similar manner, novel objects could be presented/hidden at specific times throughout the study. Such computerized phenotyping may help in elucidating the genetic determinants of behavior, pathogenic mechanisms underlying disease models, and the development of novel therapeutic strategies. If a consensus is reached with respect to testing conditions, sequence of tests, as well as the hardware and software used, one may expect that long-awaited standardization would improve reproducibility of behavioral studies and elevate experimental psychometrics to a new level.

Disclosures

Open Access publication of this article is sponsored by Med Associates, Inc. (St. Albans, VT). 


\section{Acknowledgements}

This work was supported by an Ontario Mental Health Foundation grant to B.S, and an Ontario Graduate Scholarship to M.K.

\section{References}

1. Henn, F. A., McKinney, W. T. Ch. 67. Psychopharmacology: The Third Generation of Progress . Meltzer, H. Y. 687-695 Raven Press (1987).

2. Hart, B. L. The behavior of sick animals. Vet. Clin. North Am. Small Anim. Pract. 21, 225-237 (1991).

3. Kapadia, M., Sakic, B. Autoimmune and inflammatory mechanisms of CNS damage. Prog. Neurobiol. 95, $301-333$ (2011).

4. Rogers, D. C. Behavioral and functional analysis of mouse phenotype: SHIRPA, a proposed protocol for comprehensive phenotype assessment. Mamm. Genome. 8, 711-713 (1997).

5. Moy, S. S. Mouse behavioral tasks relevant to autism: phenotypes of 10 inbred strains. Behav. Brain Res. 176, 4-20 (2007).

6. Gulinello, M., Putterman, C. The MRL/Ipr mouse strain as a model for neuropsychiatric systemic lupus erythematosus. J. Biomed. Biotechnol. 2011, 207504 (2011).

7. Marchese, M. Autoimmune manifestations in the 3xTg-AD model of Alzheimer's disease. J. Alzheimers. Dis. 39, 191-210 (2014).

8. Sakic, B. A behavioral profile of autoimmune lupus-prone MRL mice. Brain Behav. Immun. 6, 265-285 (1992).

9. Sakic, B., Szechtman, H., Denburg, S. D., Carbotte, R. M., Denburg, J. A. Spatial learning during the course of autoimmune disease in MRL mice. Behav. Brain Res. 54, 57-66 (1993).

10. Sakic, B. Disturbed emotionality in autoimmune MRL-Ipr mice. Physiol. Behav. 56, 609-617 (1994).

11. Visser, L., van den Bos, R., Kuurman, W. W., Kas, M. J., Spruijt, B. M. Novel approach to the behavioural characterization of inbred mice: automated home cage observations. Genes Brain Behav. 5, 458-466 (2006).

12. Sakic, B. The use of integrated behavioral station in chronic behavioral studies. Measuring Behavior. 328 Maastricht, Netherlands (2008).

13. Shinzawa, K. Neuroaxonal dystrophy caused by group VIA phospholipase A2 deficiency in mice: a model of human neurodegenerative disease. J. Neurosci. 28, 2212-2220 (2008).

14. Quintana, A., Kruse, S. E., Kapur, R. P., Sanz, E., Palmiter, R. D. Complex I deficiency due to loss of Ndufs4 in the brain results in progressive encephalopathy resembling Leigh syndrome. Proc. Natl. Acad. Sci. U. S. A. 107, 10996-11001 (2010).

15. Irwin, S. Comprehensive observational assessment: la. A systematic, quantitative procedure for assessing the behavioral and physiologic state of the mouse. Psychopharmacologia. 13, 222-257 (1968).

16. Crawley, J. N. What's Wrong With My Mouse?: Behavioral Phenotyping of Transgenic and Knockout Mice. Wiley-Liss (2007).

17. Feeney, D. M., Gonzales, A., Law, W. A. Amphetamine, haloperidol and experience interact to affect rate of recovery after motor cortex injury. Science. 217, 855-857 (1982).

18. Stanley, J. L. The mouse beam walking assay offers improved sensitivity over the mouse rotarod in determining motor coordination deficits induced by benzodiazepines. J. Psychopharmacol. 19, 221-227 (2005).

19. Gulinello, M., Chen, F., Dobrenis, K. Early deficits in motor coordination and cognitive dysfunction in a mouse model of the neurodegenerative lysosomal storage disorder, Sandhoff disease. Behav. Brain Res. 193, 315-319 (2008).

20. Rustay, N. R., Wahlsten, D., Crabbe, J. C. Influence of task parameters on rotarod performance and sensitivity to ethanol in mice. Behav. Brain Res. 141, 237-249 (2003).

21. Kapadia, M. Altered olfactory function in the MRL model of CNS lupus. Behav. Brain Res. 234, 303-311 (2012).

22. Jhuang, H. Automated home-cage behavioural phenotyping of mice. Nat. Commun. 1, 68 (2010).

23. Steele, A. D., Jackson, W. S., King, O. D., Lindquist, S. The power of automated high-resolution behavior analysis revealed by its application to mouse models of Huntington's and prion. Proc. Natl. Acad. Sci. U. S. A. 104, 1983-1988 (2007).

24. Zarringhalam, K. An open system for automatic home-cage behavioral analysis and its application to male and female mouse models of Huntington's disease. Behav. Brain Res. 229, 216-225 (2012).

25. Chaumont, F. Computerized video analysis of social interactions in mice. Nat. Methods. 9, 410-417 (2012).

26. Kabra, M., Robie, A. A., Rivera-Alba, M., Branson, S., Branson, K. JAABA: interactive machine learning for automatic annotation of animal behavior. Nat. Methods. 10, 64-67 (2013).

27. Weissbrod, A. Automated long-term tracking and social behavioural phenotyping of animal colonies within a semi-natural environment. Nat. Commun. 4, 2018 (2013). 